\title{
Guest editorial on S.I.: Bio-inspired computing: theories and application
}

\author{
Xinchao Zhao ${ }^{1} \cdot$ Maoguo Gong ${ }^{2} \cdot$ Xingquan Zuo $^{3} \cdot$ Linqiang Pan $^{4}$
}

Published online: 13 February 2020

c) Springer-Verlag GmbH Germany, part of Springer Nature 2020

Bio-inspired computing methodology is a fairly active research domain. This special issue on Bio-inspired Computing: Theories and Application aims at presenting the most recent research and developments in the area of bio-inspired intelligent computation based on the principles of biological, swarm and evolutionary algorithms. Briefly speaking, bioinspired computing methodology is the use of computers to model the living phenomena and the study of life to improve the usage of computers. It has attracted widely theoretical research as well as technological applications. These algorithms provide efficient tools to those problems which cannot be solved using traditional and classical mathematical methods, as the algorithms do not require any mathematical condition to be satisfied.

There are seven papers in this Special Issue. This special issue is committed to timely publication of high-quality, peer-reviewed, original articles that advance the state-ofthe art of all aspects of bio-inspired computing, evolutionary computation and swarm intelligence. It covers the

Xinchao Zhao

zhaoxc@bupt.edu.cn

Maoguo Gong

mggong@mail.xidian.edu.cn

Xingquan Zuo

zuoxq@bupt.edu.cn

Linqiang Pan

lqpanhust@gmail.com

1 School of Science, Beijing University of Posts and Telecommunications, Beijing 100876, China

2 Key Laboratory of Intelligent Perception and Image Understanding of Ministry of Education, Xidian University, Xi'an 710071, China

3 School of Computer Science, Beijing University of Posts and Telecommunications, Beijing 100876, China

4 School of Automation, Huazhong University of Science and Technology, Wuhan 430074, China state-of-the-art of bio-inspired computing methodology as well as novel theories and interesting applications.

Improved Normalized Graph Cut with generalized data for enhanced segmentation in Cervical Cancer Detection proposes a Normalized Graph Cut with Generalized Data for Enhanced Segmentation (INGC-GDES) mechanism for effective detection of the cytoplasm and nucleus boundary of the pap smear cell in an optimized manner. This proposed INGC-GDES approach is implemented over the pap smear cervix cells in order to analyze its hazy and overlapping boundaries for superior detection of cervical cancer cells. In this INGC-GDES approach, the preprocessed cervical image is converted into an improved Normalized Graph cut set for combining the merits of spatial and intensity information related to the processed image used for analysis. The method of maximum flow algorithm is applied over the derived normalized graph cut set for determining the optimal pixel points that aid in superior detection of cervical cancer.

Application of Multi-Objective Artificial Fish Swarm Algorithm in Grain Transportation Optimization Problem proposes an improved multi-objective grain transportation route optimization model based on artificial fish swarm algorithm. It is closer to the reality and is more instructive to the practical problems. Firstly, the similar segment distance is introduced into the rear-end behaviour and cluster behaviour to replace the traditional distance calculation method. Secondly, the optimal solution fragment which plays the guiding role of bulletin board in eating behaviour is inserted into the current differential solution of the bulletin board. Finally, a hybrid neighbourhood search is introduced into the three behaviours of artificial fish swarm according to the characteristics of grain transportation problem.

Prediction of forest unit volume based on hybrid feature selection and ensemble learning proposes a FL-Stacking model based on hybrid feature selection and ensemble learning according to the real data provided by the National Forestry Science Data Sharing Service Platform. It provides a scientific basis for forest resource management and 
decision-making with high dimensionality and complex samples. Firstly, the model extracts features based on FilterLasso hybrid method, then constructs the prediction model of forest unit volume based on ensemble learning. Then it uses eight prediction models, such as Linear SVM regression, as the fusion basis model in the training set by Stacking scheme. Finally, the fusion and optimization of the basic model are carried out.

Analysis of evolutionary process of fog computing system based on BA and ER network hybrid model models the evolutionary process of the fog computing node after structuring the fog computing system based on BA scale-free network and ER random network, which aims to design a safe, stable and efficient fog computing system. Then the evolutionary process of the network hybrid model is analysed. Finally, the evolutionary model of the fog computing system is solved, and the conditions that the hybrid network obeys for certain network structures in the two networks are obtained.

Application of improved time series Apriori algorithm by frequent itemsets in association rule data mining based on temporal constraint describes the working principle of the traditional Apriori algorithm and proposes an improved Apriori algorithm for frequent itemset time series. The methods and procedures of time series and time sequence association rules mining are then analyzed. A combined application of improved time series Apriori algorithm is resented by frequent itemsets in association rule data mining based on temporal constraint.

Structure Optimization Method based on Automatic Vectorization combines field storage and applies in the structure to reduce the access to these addresses during the SIMD vectorization. Structure is used more extensively to promote the performance of application program, such as scientific computing. The non-continuity and the non-aligment of vectorization structure array have a dramatic influence on the efficiency of program's vectorization. Therefore an address conversion method is proposed to map structure array one to the two dimensional array. To meet the request of the continuity and the alignment of its non-array memory address, it further reduces the failure rate of cache and improves the application performance.

A node-priority based overlapping community detection method using evolutionary multi-objective suggests a community detection method based on node priority to improve the accuracy of large-scale overlapping community detection. The proposed algorithm has two advantages. A priority function $f_{N N}$ is firstly defined to assess the closeness between adjacent nodes. It initially explores the potential community structure and reduces the scale of networks. All Pareto fronts are selected to better mine large-scale overlapping communities. Authors also employed NSGA-II to explore the community structure. Finally, the proposed algorithm is applied to the artificial and real datasets to very its performance.

We are grateful to the authors who submitted their papers to this special issue. We would also like to thank the reviewers for their hard work and their valuable feedback to the authors. Finally, we would like to express our sincere gratitude to the Editor-in-Chief for providing the opportunity and assistance to edit this special issue in the well-known journal of Evolutionary Intelligence. Without all of this, it would be impossible to organize a successful special issue dedicated to this interesting topic.

Acknowledgements This special issue is partially supported by Beijing Natural Science Foundation (1202020) and National Natural Science Foundation of China (61973042, 61873040, 71772060).

Publisher's Note Springer Nature remains neutral with regard to jurisdictional claims in published maps and institutional affiliations. 